correlation between AV45 retention and increased cortical thickness in Stage 1 subjects (FIG1). The results were similar when correlating the mean SUVR with cortical thickness. The Stage 2 group also presented a cluster of increased cortical thickness in the AV45-CTh correlation analyses. Conclusions: Brain structure in preclinical $\mathrm{AD}$ follows a biphasic trajectory of changes. This study also shows that different brain areas could be at different stages in an individual. These results have implications in clinical trials in preclinical $\mathrm{AD}$, both when selecting patients and when using MRI as a surrogate marker of efficacy.

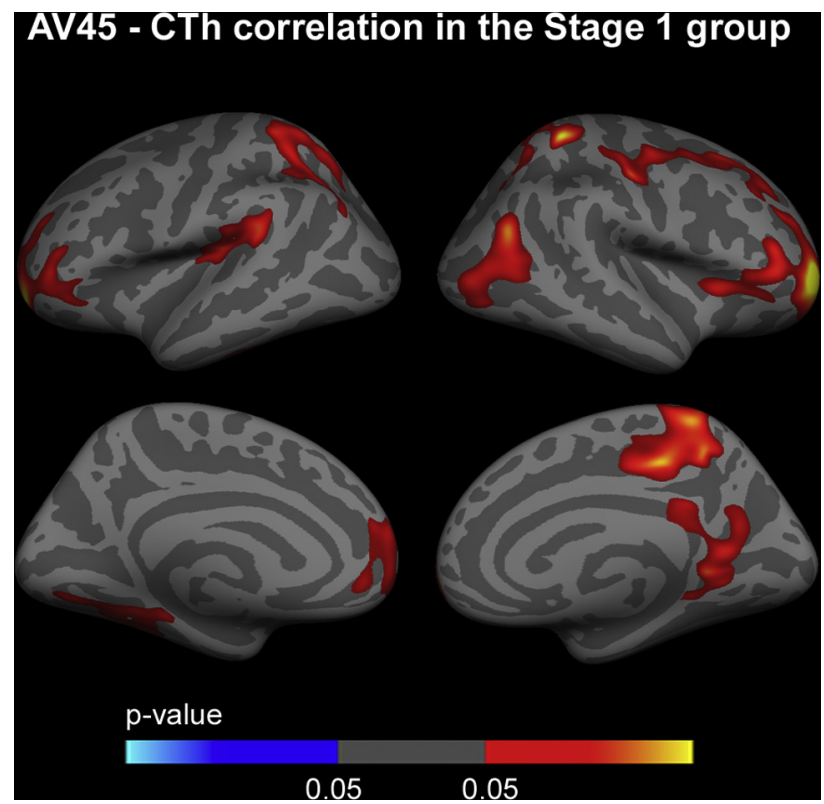

\section{O1-06-03 EFFECTS OF TAU DEPOSITION ON CEREBRAL GLUCOSE METABOLISM IN NORMAL OLDER ADULTS VARY BY AMYLOID LEVEL}

Samuel N. Lockhart ${ }^{1}$, Jenna N. Adams ${ }^{1}$, Suzanne L. Baker ${ }^{2}$, Jian Kang ${ }^{3}$, Lexin $\mathrm{Li}^{1}$, William J. Jagust ${ }^{1,2}$, ${ }^{1}$ University of California Berkeley, Berkeley, CA, USA; ${ }^{2}$ Lawrence Berkeley National Laboratory, Berkeley, CA, USA; ${ }^{3}$ University of Michigan, Ann Arbor, MI, USA. Contact e-mail: sam. lockhart@gmail.com

Background: Relationships between brain $\beta$-amyloid and glucose metabolism in cognitively normal older adults (OA) have been weak and inconsistent. We examined associations between tau and glucose metabolism and whether relationships varied with global A $\beta$ burden. Methods: 47 OA (Table) received 18F-AV-1451 (tau, 80-100 min SUVR), 11C-PiB (A $\beta$, 35-90 min DVR), and 18F-FDG (30-60 min SUVR) PET, which were coregistered to T1-weighted MRI. We processed native-space T1 (FreeSurfer v5.3) to delineate cerebellar gray matter ( $\mathrm{PiB}$ and AV-1451 reference region) and brainstem (edited to derive FDG pons reference). For each subject we calculated a weighted cortical $\mathrm{PiB}$ average to characterize A $\beta$-positivity. We warped OA MRI to MNI space (ANTS) and applied transformations to AV-1451 and FDG images. A cortical testing mask was created by intersecting AAL ROIs with high-probability gray matter (GM) voxels (SPM12 probability map). MNI-space FDG and AV-1451 PET images were masked and smoothed $(4 \mathrm{~mm})$. We used SPM12 VBM (10mm FWHM smoothing) on T1 images to generate voxelwise MNI-space GM concentration images, which were also masked. We used a newly-developed Spatially Varying Coefficient Model to examine associations between increased tau accumulation and reduced glucose metabolism (within A $\beta$ status group, controlling for local GM). This method incorporates false discovery control, accounts for continuity among adjacent voxels, and has higher detection power than voxel-wise analysis. Reported clusters are significant at $p<.0001$ ( $>=20$ voxels). Results: Among A $\beta$ - OA, increased AV1451 uptake was significantly associated with reduced FDG metabolism (Figure) in clusters located in bilateral medial temporal lobes (MTL), with additional smaller clusters in inferior frontal cortex and temporo-occipital fusiform gyrus. Among A $\beta+O A$, significant inverse $\mathrm{AV}-1451-\mathrm{FDG}$ associations were located in right inferolateral temporal, and bilateral medial parietal and inferior medial frontal cortex. Conclusions: In cognitively normal A $\beta-O A$, tau deposition is associated primarily with reduced metabolism in MTL. In A $\beta+O A$, increased tau deposition is associated with metabolic deficits in medial frontal and parietal cortex, typical of brain regions with $A \beta$ accumulation. Although the mechanisms by which late-life tau and amyloid accumulation influence glucose metabolism are not well understood, these results suggest early tau accumulation interacts with $\mathrm{A} \beta$ to produce cortical hypometabolism.

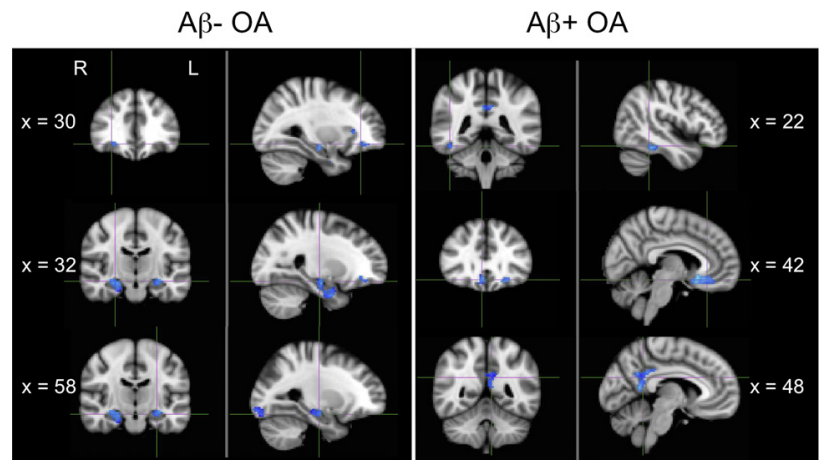

Figure. AV-1451-FDG associations by $\mathrm{A} \beta$ status. Clusters indicate significant negative associations between AV-1451 and FDG in A $\beta$ - (left) and $\mathrm{A} \beta+$ (right) older adults.

Table

Participant demographics

\begin{tabular}{lll}
\hline & $\mathrm{A} \beta-$ & $\mathrm{A} \beta+$ \\
\hline $\mathrm{N}$ & 26 & 21 \\
Age, $\mathrm{y}$ & $78.7(6.9)$ & $79.1(3.6)$ \\
Sex $(\mathrm{M} / \mathrm{F})$ & $9 / 17$ & $7 / 14$ \\
Education, y & $17.0(1.9)$ & $16.0(2.1)$ \\
Global PiB DVR & $1.01(0.024)$ & $1.37(0.24)$ \\
MMSE & $29.1(0.98)$ & $28.3(1.4)$ \\
\hline
\end{tabular}

01-06-04

\section{CSF P-TAU IS CORRELATED WITH TAU PET, WHILE A $\beta$ PET CORRELATES WITH Aß1-42 AND THE T-TAU/A $\beta 1-42$ RATIO}

James D. Doecke ${ }^{1}$, Qiao-Xin $\mathrm{Li}^{2}$, Christopher Fowler ${ }^{3}$, Steven Collins ${ }^{3}$, Vincent Dore ${ }^{4,5}$, Christopher C. Rowe ${ }^{6,7,8,9}$, Colin L. Masters ${ }^{2,6,8}$, Olivier Salvado ${ }^{10}$, Victor LL. Villemagne ${ }^{3,5,6,11}$, ${ }^{1}$ CSIRO Preventative Health Flagship, Herston, Australia; ${ }^{2}$ The Florey Institute of Neuroscience and Mental Health, Parkville, Australia; ${ }^{3}$ The Florey Institute of Neuroscience and Mental Health, Melbourne, Australia; ${ }^{4}$ CSIRO, Melbourne, Australia; ${ }^{5}$ Austin Health, Melbourne, Australia; ${ }^{6}$ AIBL Research Group, Perth and Melbourne, Australia; 